\title{
Novelar la comedia: de la inserción de la trama matrimonial teatral en la novela inglesa dieciochesca
}

\section{Comedy Goes Novel: Of the Inception of the Theatrical Marriage Plot in the Eighteenth-century Novel}

\author{
ANAGLARA CASTRO-SANTANA \\ Facultad de Filosofía y Letras \\ UniVERSIDAD NAGionAL AUTÓNOMA DE MÉXico | México \\ Contacto: anaclaracastro@filos.unam.mx
}

\section{Resumen}

Un fenómeno curioso y digno de estudio puede observarse entre mediados del siglo XVIII y la primera mitad del XIX: la prevalencia de la trama matrimonial en la novela británica. La obra de Jane Austen salta de inmediato a la imaginación cuando se piensa en el matrimonio como fin diegético y motor narrativo en este periodo histórico. Sin embargo, si bien es con esta autora que la trama de cortejo llega a una cúspide en la que el uso serio e irónico convergen, los inicios de su cimentación como fórmula narrativa favorita pueden rastrearse a la segunda mitad del siglo XVIII. En este artículo se propone una hipótesis para esta observación: que, tras la aprobación del Acta de Licencia Teatral de 1737, el relativo estancamiento de la comedia teatral (en la que imperaba la trama matrimonial desde el periodo de la Restauración) fue decisivo para el florecimiento de la novela. Cuando el teatro, que solía ser tanto la profesión literaria más redituable, como el medio de entretenimiento masivo más socorrido, cedió terreno a la narrativa en prosa, la trama por excelencia de la comedia teatral se convirtió en el modelo narrativo de elección para los novelistas. 


\title{
Palabras clave
}

trama matrimonial, teatro y novela, narrativa anglófona dieciochesca, relaciones intergenéricas, final feliz

\begin{abstract}
An intriguing phenomenon can be observed between the second half of the eighteenth century and the first of the nineteenth: the extensive use of the marriage plot in mainstream British novels. Jane Austen is probably the name that comes to mind when thinking about the marriage finale as the point in which diegetic closure and narrative structure neatly converge. Looking back to the second half of the eighteenth century might yield a different angle on the story, however. Although Austen takes the marriage finale to its peak by resorting to it seriously and ironically at the same time, the inception of the marriage plot in the novel can be traced back to the second half of the century. Stage comedy had adopted the marriage plot as a tacit norm since at least the Restoration. By the eighteenth century, it was so widespread as to warrant mockery. This article teases the hypothesis that it was the flourishing of the novel, fostered by the relative decay in theatrical innovation at a time when the theatre was the favourite literary medium for widespread instruction and entertainment, that promoted the introduction of the marriage plot into the novel.
\end{abstract}

\section{Keywords}

marriage plot, theatre and novels, eighteenth-century fiction, intergeneric relations, happy ending

\footnotetext{
Al final de Emma (1816) de Jane Austen, la protagonista homónima se casa con Mr. Knightley; ante ellos se extiende el prospecto de "felicidad absoluta" que satisface las expectativas e ilusiones de sus allegados más queridos (Austen, 2000: 319). ${ }^{1}$

1 "The wishes, the hopes, the confidence, the predictions of the small band of true friends who witnessed the ceremony were fully answered in the perfect happiness of the union" (Austen, 2000: 319).
} 
Su unión sucede tras haber superado una serie de obstáculos y malentendidos que dan lugar a reflexiones en torno a las costumbres sociales, las cuales constituyen el verdadero interés de la lectura. Si bien, a la manera de los relatos de misterio, la identidad del contrayente ideal para la heroína se mantiene en una suerte de secreto que se va descubriendo poco a poco, tras desechar a otros candidatos cuyas fallas se descubren de manera paulatina, el enlace matrimonial es esperado desde el inicio de la historia. Algo similar ocurre en las otras cinco novelas de la autora: Sense and Sensibility (1811), Pride and Prejudice (1813), Mansfield Park (1814), Northanger Abbey (1817) y Persuasion (1818). Lo mismo sucede en novelas anteriores, como Evelina (1778) y Cecilia (1782) de Frances Burney, foseph Andrews (1742) y Tom fones (1749) de Henry Fielding, el volumen original de David Simple (1744) de Sarah Fielding, Pamela (1740) y Sir Charles Grandison (1753) de Samuel Richardson, por mencionar algunos ejemplos famosos. Con frecuencia, en este tipo de narrativas, con sólo ver la cubierta del libro y hojear las primeras páginas, se puede saber que uno o más matrimonios darán conclusión a la historia principal y que serán felices durante el tiempo diegético restante entre el anuncio o la celebración de su matrimonio y el punto final del libro. Esta expectativa obedece tanto al género literario al que pertenecen, como al momento histórico y a la locación geográfica de su producción - en pocas palabras, a su condición como novelas domésticas, o novelas de cortejo, de los albores de la modernidad inglesa-. Esta terminología será precisada más adelante; sin embargo, para fines prácticos, en este artículo utilizaré las frases "novela de cortejo" y "novela doméstica”, así como "trama matrimonial” y "trama de cortejo" de forma intercambiable, excepto cuando sea necesario aclarar sus diferencias más puntuales.

Si bien el amor romántico ha sido uno de los grandes focos filosóficos y estéticos de la literatura a lo largo del tiempo, la conclusión de éste en un matrimonio que funja como cierre para un relato no es, de manera alguna, universal. Ocurre con cierta frecuencia en el género narrativo del romance, el cual puede rastrearse desde la tradición grecolatina - siendo Dafnis y Cloe (s. II d. c.) de Longo el ejemplo más famoso - con auge en la Europa medieval, momentos de repunte a lo largo de los siglos XVI y XVII, hasta su absorción y reemplazo gradual por la novela moderna 
(McDermott, 1989). ${ }^{2}$ En la narrativa anglófona en prosa, el final matrimonial sucede de manera intermitente en romances y novelas, desde que se tiene registro hasta nuestros días, pero difícilmente puede hablarse de una presencia constante en estas últimas, salvo en el periodo comprendido entre la segunda mitad del siglo XVIII y el primer cuarto del XIX. De modo curioso, pero no casual, este mismo momento histórico se caracteriza por la elevación cultural de la novela y su consolidación como género literario dominante en Inglaterra (Warner, 1998).

A lo largo del tiempo, se ha estudiado la influencia del romance y otros géneros populares como los manuales de conducta, los relatos de viajes, los ensayos periódicos, las biografías espirituales y las biografías criminales en el desarrollo de la novela moderna en lengua inglesa (Hunter, 1990; Doody, 1996; McKeon, 2000). Una de las características principales de la novela es su maleabilidad, así como su capacidad para absorber y sintetizar otros géneros discursivos. Como bien apunta Patricia Meyers Spacks (2006), las novelas dieciochescas pueden entenderse como una serie de experimentos literarios. Una de las áreas menos estudiadas del fenómeno del afianzamiento de la novela como forma narrativa preponderante de la modernidad tiene que ver con el desplazamiento del teatro por la novela como fuente innovadora de entretenimiento y medio masivo de instrucción. Todo esto cobra relevancia particular si se tiene en cuenta que en el teatro, a diferencia de lo que ocurre con la narrativa en prosa, la trama de cortejo es una estructura mucho más duradera, que puede observarse con regularidad desde, al menos, la así llamada comedia nueva griega, que forma la base de la shakespeariana, cuya influencia $-\mathrm{y}$

${ }^{2}$ La influencia del romance en la novela anglófona es un fenómeno bien documentado. McKeon (1987) explica sus diferencias en términos de especialización, la cual, considera, está ligada a la división del trabajo y la separación del conocimiento en los umbrales de la época moderna en Inglaterra. Hay quienes han llegado al extremo de afirmar que no hay diferencia alguna entre la novela moderna y el romance de antaño, como lo hace Margaret Doody (1996) en su controvertido estudio The True History of the Novel. Allí, Doody argumenta, con todas sus letras, que "Romance and the Novel are one" (15), aunque los ejemplos que utiliza para ilustrar la continuidad temática entre los géneros dejan ver importantes diferencias formales y discursivas. En un texto más reciente, David H. Richter (2017) explora de manera detallada los puntos de contacto y las disimilitudes entre romances y novelas, así como los cambios en su público meta a lo largo del siglo XVIII. El propósito del presente artículo no es negar u oscurecer la afiliación entre la novela y el romance, sino abonar al entendimiento de las dinámicas entre los géneros literarios de los albores de la modernidad, por medio del reconocimiento del teatro como un modelo novelístico poco atendido por la crítica. 
con el tiempo preponderancia - determina las obras del repertorio teatral dieciochesco. ${ }^{3}$ Tomando estas aseveraciones como punto de partida, en este artículo me daré a la tarea de explorar uno de los posibles caminos que llevaron a la popularización del matrimonio como el desenlace ideal esperado en la novela dieciochesca inglesa a partir de la segunda mitad del siglo XVIII: la importación y adaptación de la trama matrimonial - en particular en su vertiente de cortejo- de la comedia teatral a la novela.

La historia de los géneros literarios es un tema fascinante, de una extensión descomunal imposible de abarcar o resumir en un breve artículo de ambiciones limitadas como éste. Así pues, las siguientes páginas no pretenden, de modo alguno, ser un ejercicio exhaustivo, ni proveer una explicación unívoca y reduccionista, sino presentar un cuestionamiento sugerente sobre un fenómeno particular y poco atendido por la crítica que tuvo lugar en Inglaterra en el momento en que la narrativa ficcional en prosa, que a la postre se denominó novela, estaba en proceso de consolidarse como género literario de prestigio. Comenzaré por abordar de manera sucinta las convenciones de la comedia matrimonial en el teatro dieciochesco, incluyendo las formas en que se tornaban objeto de ridículo durante las primeras décadas de aquella centuria. Al centro de esta breve relación se situará el Acta de Licencia Teatral de 1737, que marca un hito en el dinamismo y la originalidad de la dramaturgia del periodo en cuestión. Procederé a atender las contribuciones al género novelístico por parte de Fielding y Richardson, dos grandes prosistas de mitades del siglo XVIII, a quienes con frecuencia se estima pioneros en la campaña por la legitimación cultural de la novela, el primero de ellos dramaturgo de corazón y el segundo, antagonista acérrimo del teatro. Ambos escritores echan mano de la trama matrimonial y sus obras más famosas desde el inicio se consideraron parteaguas para el desarrollo de la novela. Abordaré esta historia ya

${ }^{3}$ El término "nueva comedia griega" hace referencia al tipo de tramas popularizadas por Menandro que, en contraste con la "vieja" comedia aristofánica, privilegian las historias de amor y las intrigas domésticas con personajes cotidianos. Como lo explica Oliver Taplin (2001), en la nueva comedia "the plots almost always involve a love story which ends, after problems, with boy-marriesgirl" (43). El vínculo de la comedia shakespeariana con la "nueva comedia" se abordará de manera más amplia en la siguiente sección de este artículo. 
conocida desde un ángulo poco tratado: su relación con la dramaturgia. Concluiré con una breve reflexión sobre la consolidación de la trama matrimonial, con sus acendrados tintes irónicos, en Austen, y el posicionamiento aún más abierto contra ésta que puede observarse en un par de novelas decimonónicas - Fane Eyre (1847) de Charlotte Brönte y Wuthering Heights (1847) de Emily Brönte- en las que se trastoca la convención y se juega con las expectativas de los lectores, de manera similar a lo que sucedía en la comedia teatral británica durante las primeras décadas del siglo XVIII.

\section{De lugares comunes, subversiones y censuras}

El año 1737 se considera aciago para la dramaturgia anglófona. El 21 de junio entró en vigor una ley que cambió la cara del teatro en Gran Bretaña en los años y décadas por venir: el Acta de Licencia Teatral (Thomas, 2014: 96). Esta regulación estipulaba que ninguna obra nueva podía ser puesta en escena sin pasar antes por el escrutinio meticuloso del Lord Chambelán y que sólo los teatros poseedores de una patente otorgada por la corona - Drury Lane y Covent Garden en Londres, más los teatros reales ubicados en provincias como Bristol, Norwich y York- estarían autorizados para presentar y cobrar por funciones teatrales. ${ }^{4}$ Los puntos principales de interés para el censor, que hacían a una obra acreedora a correcciones o a prohibición total, eran, en orden de importancia, la sedición política, el descaro sexual y la transgresión de normas sociales en general.

De acuerdo con el consenso crítico, esta ley -que se mantuvo en vigor hasta 1968 - tuvo como consecuencia principal una caída en la innovación y el dinamismo del teatro británico (Thomas, 2014: 99; Milhous, 2004: 125; Hume, 1988: 249250). Casi de la noche a la mañana, la dramaturgia se volvió una profesión muy poco redituable. A juicio de Matthew J. Kinservik (2002), "the prohibition of the

${ }^{4}$ Es interesante notar que algunos dueños de teatros alternativos lograban saltarse la regulación - al menos por un tiempo- mediante trucos (por ejemplo, puestas en escena que se decían gratuitas, pero en las que se cobraba por bebidas o alimentos obligatorios) o aprovechando vacíos legales (por ejemplo, abriendo pequeños teatros en los suburbios londinenses que estaban fuera de la jurisdicción del Lord Chambelán) (Moody, 2012). 
non-patented theatres and the conservative repertories of Drury Lane and Covent Garden made earning a living as a playwright all but impossible. Staging a new play was an increasingly costly and troublesome venture” (106). Esto se manifiesta con mayor rigor en la comedia y en los primeros cincuenta años después de la implementación del Acta de Licencia Teatral. Con el tiempo y la práctica, tanto autores como espectadores se acostumbraron a la censura, de suerte que las obras “arrive[d] at the Lord Chamberlain's office already corrected by playwrights who had internalised the new standards" (Kinservik, 2002: 11). La aclamada recepción hacia la década de 1770 de comedias inteligentes y graciosas, pero más recatadas y con la corrección política interiorizada, como She Stoops to Conquer (1773) de Oliver Goldsmith, The Rivals (1775) y The School for Scandal (1777) de Richard Sheridan es muestra clara de esta aseveración. ${ }^{5}$

El Acta de Licencia de 1737 también trajo consigo el auge de la profesión actoral, dado que, ante la limitación en la producción de nuevas obras, se esperaba que la representación aportara la innovación que hacía falta (Thomas 2014: 102). Un vistazo a los anuncios de las puestas en escena -que se publicaban en los diarios londinenses - revela el papel preponderante que tenía para el público teatral la confección de nuevas escenografías, la adición de números musicales, así como la participación de las estrellas del momento en obras bien conocidas (a la manera en que en la actualidad una nueva producción de Mujercitas con Emma Watson acerca un clásico a espectadores más jóvenes). Los dramatis personae sugieren también algunas dinámicas de actuación que le daban interés adicional a la representación. Por ejemplo, a parejas de actrices y actores cuyo involucramiento romántico era bien conocido por el público, con frecuencia se les asignaban papeles en los que debían manejar tramas de adulterio, de cortejo o de rivalidad, lo cual potenciaba el efecto cómico o de tensión de la puesta en escena. ${ }^{6}$ Las nuevas

${ }^{5}$ Aun así, The Rivals recibió abucheos en la noche de su estreno debido a lo que algunos espectadores consideraron como bromas de mal gusto contra la milicia y los irlandeses.

${ }^{6}$ Este tipo de materiales paratextuales fue compilado y estudiado por primera vez en conjunto en los cinco volúmenes que componen The London Stage, 1660-180o: A Calendar of Plays, Entertainments \& Afterpieces, together with Casts, Box-receipts and Contemporary Comment; Compiled from the Playbills, Newspapers and Theatrical Diaries of the Period (1960-1968). En la actualidad, 
circunstancias también contribuyeron a la cimentación del culto shakespeariano, puesto que los dueños de las compañías teatrales autorizadas por la corona recurrieron más que nunca a clásicos de éxito probado y Shakespeare se encontraba en proceso de erigirse como símbolo por excelencia de lo británico (Ritchie y Sabor, 2012: 1-4). Otra consecuencia insospechada - la de mayor interés para el argumento que aquí se presenta - fue el aumento en el alcance de la novela como género literario destinado tanto al entretenimiento como a la instrucción de las masas. Tras la aprobación de la ley, muchos dramaturgos perdieron su empleo. Como argumenta Richard W. Bevis (1988), "dramatic talent was diverted elsewhere” (194). Y ese otro sitio, en la mayoría de las ocasiones, fue la narrativa en prosa. Para algunos, entre ellos Henry Fielding, el comediógrafo más fructífero y prometedor de su momento, la dramaturgia, además de un ingreso, representaba la posibilidad de adquirir fama y transmitir ideas, con la ambición de generar cambios sociales. Desde principios de siglo, la narrativa en prosa ya había probado ser un medio lucrativo en Inglaterra: el gran éxito comercial de Gulliver's Travels de Jonathan Swift y de las obras de Eliza Haywood y Daniel Defoe hablaba por sí mismo. Sin embargo, la novela todavía era un género tildado de menor, en el mejor de los casos, y asociado con el escándalo y la ignominia, en el peor de éstos (Warner, 1998: 1-5). No obstante, como lo manifiesta Deidre Lynch (2019), una de las características de la cultura dieciochesca es su avidez por conferir "power to writing as a technology of world making” (22). Entonces, mientras que en el teatro el freno a la innovación y el énfasis en la representación dieron lugar al auge de la espectacularidad, la novela poco a poco se apropió del ejercicio intelectual por medio de la palabra. No es de extrañar, entonces, que pronto fuera reevaluada y vista como el medio idóneo para educar a las masas.

Antes de dar el salto hacia el género novelístico, sin embargo, conviene ir un poco atrás en el tiempo para considerar la importancia de la trama matrimonial en el teatro británico. En 1714, el dramaturgo satírico John Gay, amigo y colabo-

puede consultarse en la base de datos de acceso gratuito The London Stage Database: https:// londonstagedatabase.usu.edu/ 
rador de Swift y Alexander Pope, ponía en evidencia lo que consideraba como los grandes defectos del teatro de su momento: la mezcla de géneros y tonos, ligados a la prevalencia ad nauseam de la trama matrimonial en la comedia teatral. Su The What D'Ye Call It? A Tragi-Comi-Pastoral Farce, consiste en una colección de peripecias inconexas y una trama que serpentea hasta llegar al casamiento de los protagonistas del ensayo de una obra teatral que se desarrolla dentro de la principal. Este matrimonio ocurre a instancias de Sir Roger, director de la obra dentro de la obra, quien clama: "what's a Play without a Marriage? and what is a Marriage, if one sees nothing of it?” (Gay, 1983: 180). Su necedad y propensión al cliché le cuestan su peculio, puesto que el matrimonio que se representa en escena, entre su hijo terrateniente y una campesina, a final de cuentas resulta real, lo cual deviene una caída de estatus para su familia. ${ }^{7}$

La broma con que Gay cierra su farsa pone de relieve la demanda que había en aquel momento por tener finales felices en todo tipo de piezas teatrales (incluyendo tragedias), así como la costumbre de que el único final feliz posible fuera la celebración (o al menos el anuncio) de uno o más matrimonios. En palabras burlonas de George Farquhar (1988), otro dramaturgo cómico de los albores del siglo XVIII, "as the Catastrophe of all Tragedies is Death, so the end of Comedies is Marriage" (81). Esta práctica puede rastrearse al momento en que la comedia shakespeariana comenzó a volverse el modelo preferido de este género teatral en Gran Bretaña, es decir, el periodo de la Restauración (1660-1688). ${ }^{8}$ Como lo explica Misty Anderson (2002):

the most likely promise made to the audience in that generic contract after Shakespeare is the guarantee of a play that culminates in a marriage

${ }^{7}$ Antes de la promulgación del Acta de Causas Matrimoniales de 1754, existían discrepancias legislativas que permitían que una promesa de matrimonio formulada en tiempo presente y frente a testigos tuviera valor legal (Stone, 1990 [1979]: 30-33).

${ }^{8} \mathrm{Si}$ bien en ocasiones el periodo de la Restauración llega a extenderse hasta 1700, en términos teatrales se hace un corte tras la llamada Revolución Gloriosa, la cual cementala sucesión protestante y marca una nueva era en términos de disminución del poderío monárquico, desmitificación del derecho divino y la devaluación del monarca desenfadado y parrandero, ligado en la comedia teatral a la figura del libertino. 
that affirms the community. [...] After Shakespeare, most writers of stage comedies turned to the Greek New Comedy for their plots, which placed a greater emphasis on courtship and marriage than Aristophanic, satiric, or Jonsonian comedies had. Imperfect as this description is, it identifies the narrative thrust of most stage comedies in Restoration and eighteenthcentury England. (9)

Así, mientras que los dramaturgos trágicos de la Restauración tomaron como modelo principal el neoclasicismo francés - para el que Shakespeare resulta una anomalía - los cómicos dieron cada vez más peso a la trama matrimonial, alejándose, al menos en apariencia, de los temas abiertamente políticos. Ya entrado el siglo XVIII, la trama doméstica siguió siendo una constante, pero los personajes principales de la alta burguesía comenzaron a reemplazar a los de la aristocracia y el libertinaje fue perdiendo terreno ante la domesticidad (Bevis, 1988: 117-119). Dramaturgos como Gay continuaron usando la trama matrimonial de manera burlesca y satírica, como sucede en su famosa Beggar's Opera (1728), en la que las alianzas amorosas, el bajo mundo de las cárceles y los ladronzuelos se equiparan con las prácticas de la clase gobernante.

Algunos más continuaron echando mano de la trama matrimonial como un vehículo conveniente para transmitir crítica social. Esto puede observarse en escritores políticos como Fielding, y también en dramaturgas con agendas más discretas como Susannah Centlivre, Eliza Haywood, Hannah Cowley y Elizabeth Inchbald. Como sostiene Anderson (2002), sus obras "are balanced between the foregone conclusions of genre and the possibility for innovation” (44). Muchas veces, esa suerte de contrato pre-establecido entre la obra y los espectadores - de tener un final feliz en el que una o más parejas se casaran- resultaba liberador, puesto que permitía a los autores dedicar toda su atención a construir las negociaciones que llevaban al matrimonio (Anderson, 2002: 45-71). De manera similar, el público podía disfrutar de la intriga y el suspenso, a sabiendas de que al terminar la tormenta siempre habría de formarse el arcoíris de la unión matrimonial. 
En este punto, considero importante hacer una breve digresión para precisar el término "trama matrimonial”, utilizado con frecuencia en textos críticos, pero pocas veces definido. Hay quienes la entienden como una historia "that ends, or almost ends, in a marriage or marriages, and is largely concerned throughout with courtship" (O’Connell, 2011: 384). Sin embargo, esta definición acota demasiado el concepto, pues deja de lado las tramas que tienen que ver con la resolución de altercados entre parejas ya casadas. A este tipo, algunos le llaman de conflicto doméstico (domestic distress), en donde el adulterio es el motivo más frecuente (Boone, 1987: 66-68; Tanner, 1979: 4-13). Cuando la historia orbita en torno a parejas de contrayentes y termina en un matrimonio, se le conoce como trama de cortejo, la cual forma parte de la trama matrimonial. En ocasiones, estos últimos dos términos se utilizan como sinónimos. En el terreno de la narrativa, ambas estructuras se subsumen dentro de la novela doméstica (domestic fiction o domestic novel), término que, para algunos, es un subgénero específico de la novela decimonónica (Freedgood, 2006), pero que también designa relatos tanto dieciochescos como decimonónicos que tratan situaciones del ámbito privado - en especial el matrimonio y la familia - y se relacionan de manera tangencial con lo público - como la política nacional e internacional- (Arac, 2019; Armstrong, 1987).9 En tanto que mi trabajo tiende puentes entre la dramaturgia y la narrativa, prefiero utilizar el término trama matrimonial, o trama de cortejo, cuando es preciso poner de relieve que la historia se encamina hacia el matrimonio como final feliz.

Cierro el paréntesis terminológico para volver a la comedia teatral en la primera mitad del siglo XVIII. Fue en este contexto, en que la trama de cortejo reinaba suprema, que la innovación dramática se vio coartada por el Acta de Licencia Teatral de 1737. Tres años más tarde, justo en el momento en que otrora dramaturgos como Fielding buscaban nuevos horizontes literarios y autoras que habían experimentado con la dramaturgia, como Haywood, regresaban a la prosa, Richardson sorprendió al mundo anglófono con Pamela; or Virtue Rewarded (1740). ${ }^{10}$ El libro no

${ }_{9}^{9}$ También hay quienes le llaman novela doméstica (domestic fiction) a una categoría particular de novelas publicadas entre 1830 y 1870 en Estados Unidos (Forcey, 2005).

${ }^{10}$ Para ganarse la vida Fielding buscó licenciarse como abogado; para seguir teniendo una voz literaria en la esfera pública, se dedicó a escribir ensayos político-literarios en The Champion, una publicación periódica que él mismo fundó, y otras publicaciones similares (Downie, 2009: 83-85). 
tardó en convertirse en un fenómeno mediático, con traducciones a otros idiomas y otros medios literarios y no literarios que van desde la poesía hasta los juegos de cartas (Keymer y Sabor, 2005: 48-49). Pamela no sólo resultó popular entre el público lector de novelas, sino también fue admirada en círculos religiosos e intelectuales (Keymer y Sabor, 2005: 23-25). En formato epistolar, esta novela presenta la historia de una joven sirvienta que resiste los intentos de conquista, o más bien de abuso, de su empleador, quien, tras leer a hurtadillas la correspondencia entre Pamela y sus padres, se da cuenta del valor moral e intelectual de ella y le propone matrimonio. Esta unión, por medio de la cual la empleada doméstica se transforma en la señora de la casa, constituye la recompensa a la que hace referencia el título completo de la novela. Resumido de esta manera, el relato resulta sin duda disonante a oídos modernos, por su forma de vincular el matrimonio con el abuso (sexual y de poder), así como su tendencia a glorificar la sexualidad/ virginidad como moneda de cambio para subir en el escalafón social. Sin embargo, Pamela también ha sido leída como una novela que reconoce el valor moral, la individualidad y la sensibilidad de una mujer de clase trabajadora, algo muy poco frecuente en la literatura de la época (Keymer, 2001: xxii). Para Armstrong (1987), una de las teóricas de la novela dieciochesca y decimonónica más influyentes, al naturalizar el progreso social por medio del matrimonio, Pamela ilustra una de las características principales de la novela doméstica, puesto que encara las teorías contractuales de su momento y las rearticula, para mostrar que el contrato sexual puede entrelazarse con (e incluso imperar sobre) el contrato social (35-48). En otras palabras, por medio del enlace matrimonial entre mujeres cuya dote no fuera económica sino moral y hombres poseedores de las características inversas, la aristocracia en decadencia podía ser rescatada.

Como lo he abordado de manera más detallada en publicaciones anteriores, desde el punto de vista formal, Pamela sigue muy de cerca las convenciones de la trama de cortejo - que, como hemos visto, era la norma tácita de la comedia teatral- excepto en un punto: la narración se prolonga más allá de la boda, para mostrar la manera en que la nueva pareja se adapta a su círculo social (Castro-Santana, 2018: 107-127). Fielding fue el primero de una hueste de autores y autoras en 
reaccionar ante Pamela por medio de la escritura creativa, en un afán de participar de las ganancias económicas y del capital cultural que, gracias al éxito crítico de esta novela, comenzaba a vislumbrarse como posibilidad para la narrativa en prosa. Fielding retoma la historia primero en una parodia directa con Shamela y más tarde en una suerte de corrección indirecta con foseph Andrews, donde comienza a articular su teoría narratológica. En esta última, Fielding (1967) presenta el concepto de "comic epic poem in prose" para hablar de las características formales del tipo de relato que ambicionaba con establecer como nuevo paradigma (4). En el prefacio a esta novela, Fielding insinúa que, dado que entre los textos clásicos no se conserva ningún ejemplo de epopeya cómica, él tomará como modelo la comedia teatral (1967: 3). De manera muy similar a este género, foseph Andrezs termina con el anuncio del matrimonio entre el protagonista y su novia de toda la vida, el cual se ve venir desde el principio. Fielding repite un esquema similar en su siguiente novela de larga extensión, considerada su obra maestra y la que consolidó su fama como creador de "A New Species of Writing” (An Essay..., 1751): Tom fones (1749). ${ }^{11}$ En esta novela, el protagonista homónimo debe sortear todo tipo de obstáculos para probar su valor moral, descubrir su linaje perdido y así ser digno de desposar a su vecina y amor de infancia. El matrimonio ocurre en el capítulo final. En una suerte de epílogo, se anuncia la prosperidad de la que gozó la feliz familia en los años venideros.

De acuerdo con la historia literaria tradicional, la creatividad auspiciada por la rivalidad entre Richardson y Fielding los hizo trascender como figuras clave en la legitimación cultural del género novelístico, el primero moral y el segundo intelectual (McKeon, 2000: 623). Un factor importante, que no ha sido estudiado lo suficiente, es la postura de estos escritores y de su público lector ante el teatro. Si transportáramos el escenario que he tratado de presentar en estas páginas a la época actual, la idea de que la televisión, el cine y las plataformas electrónicas con contenidos multimedia influyen no sólo en la temática sino también en la estructura narrativa de las novelas que se publican en nuestros días sería una obviedad. No obstante, son pocos los trabajos críticos dedicados a estudiar el fenó-

${ }^{11}$ Antes de Tom fones, publicó fonathan Wild (1743) y The Female Husband (1746), dos relatos que utilizan la trama matrimonial en su vertiente de conflicto doméstico. 
meno equivalente en el siglo XVIII. El teatro y la novela de mediados del siglo XVIII compartían a sus lectores y espectadores. Muchos autores y autoras incursionaban en ambas modalidades literarias, en especial durante la segunda mitad del siglo, cuando la novela era un poco más respetable y la dramaturgia un poco más inaccesible. Haywood, por ejemplo, la novelista más famosa de la primera mitad del siglo XVIII, apodada por Fielding (2004) como "Mrs. Novel" en honor a su realce en aquel género (215), también estuvo involucrada mucho tiempo en el medio teatral. ${ }^{12}$ Fielding continuó intentando ser dramaturgo después de 1737. Falló en todas las ocasiones, en gran medida porque su interés en temas sexuales transgredía el ethos favorecido por el Acta de Licencia Teatral (Kinservik, 2002: 112-116). Frances Burney, quien, en el prefacio a Evelina, introduce una disertación narratológica en la que ensalza el legado conjunto de Richardson y Fielding para el género novelístico, también incursionó en el teatro, pero con mucho menor gloria. Mientras que sus novelas fueron muy bien recibidas, su comedia The Wittings, escrita en 1779, fue publicada por primera vez en 1994 y puesta en escena en 1998 (Rogers, 2010). Algo similar ocurrió a Frances Sheridan (madre de Richard Sheridan), quien se volvió famosa por su magistral novela Sidney Bidulph (1761), pero sólo con mucho esfuerzo y la influencia de su esposo, el actor Thomas Sheridan, y su amigo David Garrick, dueño de Drury Lane, logró ver dos de sus comedias teatrales representadas (Ross, 2004). ${ }^{13}$

${ }^{12}$ Por no hablar de Aphra Behn (1640-1689) y Catharine Trotter (1679-1749), quienes brillaron por igual como dramaturgas y como novelistas. Behn, sin embargo, murió mucho antes del periodo histórico que concierne a este artículo y Trotter, habiendo migrado al norte de Escocia desde la década de 1720, había abandonado la escena literaria para el momento que nos ocupa. Son cuantiosas las novelistas famosas de la primera mitad del siglo XVIII que también escribieron teatro e intentaron posicionarse como dramaturgas. Entre ellas, cabe mencionar a Delarivière Manley, la afamada autora de The New Atalantis (1709) y otras novelas políticas, quien en los inicios de su carrera escribió una comedia y un par de tragedias teatrales, las cuales le acarrearon acerbas críticas que la llevaron a enfocarse de lleno en la narrativa. Con la notable excepción de Susanna Centlivre, durante las primeras décadas del siglo XVIII, la mayor parte de las escritoras tuvieron mucho más éxito como novelistas que como dramaturgas, dado que la industria del libro se regía por lo que vendía bien, mientras que la escena teatral, desde antes del Acta de Licencia, estaba regulada por el gusto (bueno o malo) y los (múltiples) prejuicios de los dueños de las compañías teatrales.

${ }^{13}$ Un fenómeno opuesto puede observarse décadas más tarde con Elizabeth Inchbald y Hannah Cowley, quienes supieron aprovechar y moldear el gusto popular por la denominada comedia sentimental y cosecharon éxitos como dramaturgas. Inchbald también escribió dos novelas que gozaron de buena popularidad en su momento. 
Richardson, por su parte, estaba en contra del teatro (Warner, 1998: 224226). En Pamela trata de distanciarse de lo teatral, como una forma de destacar la respetabilidad de la protagonista y de la novela misma (Michals, 2010: 193). En Clarissa (1747-1749), invoca al teatro como modelo negativo. Tanto el nombre como las acciones de Lovelace, el villano de la historia, recuerdan a uno de los libertinos teatrales más famosos de la época, Loveless, héroe en desgracia de Love’s Last Shift (1695) de Colley Cibber y su secuela, The Relapse (1696) de John Vanbrugh, un par de obras que continuaban formando parte importante del repertorio a cincuenta años de su estreno. Lo que es más, en Clarissa, Richardson niega a sus lectores la satisfacción del final matrimonial feliz, a pesar de las numerosas cartas que le escribían sus admiradores pidiéndoselo (Barbauld, 1804: cx-cxi). ${ }^{14}$ En vez de esto, opta por un desenlace funesto, que de manera irónica resuena con otro género teatral, la tragedia - más específicamente, la denominada she-tragedy, tan socorrida en la primera mitad del siglo XVIII-. ${ }^{15}$ Para su tercera novela, Sir Charles Grandison (1753), Richardson regresa al final matrimonial, esta vez de la misma manera que en las comedias teatrales, expedito, contundente y exultante.

En resumidas cuentas, partidarios del teatro o no, los novelistas dieciochescos se posicionaban de uno u otro modo ante la forma de entretenimiento y manifestación literaria más popular de su momento. Así, la trama matrimonial, que había poblado los escenarios durante décadas, se insertó en la novela cuando ésta se afianzaba como género literario de prestigio. Desde luego, antes del Acta de Licencia Teatral, muchas novelas trataban temas amorosos (las cuantiosas obras de Haywood, Behn, Delarivière Manley, Penelope Aubin, Mary Davis dan cuenta de ello). Esas historias, no obstante, rara vez concluyen en un matrimonio feliz. Como observa Lynch (2019),

${ }^{14}$ Clarissa fue publicada en tres entregas. Es preciso notar que la decisión de Richardson de negar el final feliz es coherente con el eje moral de la novela y también constituye una respuesta a las críticas que se le habían hecho a Pamela, novela en que la protagonista termina desposando a un personaje que a lo largo de la obra la acosa sin cesar. En Clarissa, una vez que la protagonista es violada por el villano, ya no hay vuelta atrás en el desenlace fatídico.

${ }_{15}$ "She-tragedy" es el término acuñado por el dramaturgo Nicholas Rowe, en el prefacio a fane Shore (1714), para designar el tipo de tragedias que lo llevaron a la fama. Se trata de obras cuya trama gira en torno al error fatídico de su heroína y que, por lo general, concluyen con la muerte de ésta y, en ocasiones, la restauración de su reputación. 
especially in that early eighteenth-century era when liberalism was shiny, new, and strange, there remained a plurality of narrative paradigms of female subject formation-alternative accounts, that is, of how consent to exchange might function as an act of self-inscription producing individual personhood and voice. (24)

Muchos de los relatos de ese periodo terminan con la muerte (social o física) de las heroínas, tras la incidencia de embarazos no deseados que revelan al mundo su sexualidad descontrolada, o con emparejamientos no heteronormativos que les traen reposo después de una juventud de engaños y decepciones causadas por la normativa patriarcal. ${ }^{16}$

\section{Consideraciones finales, o del afianzamiento y resquebrajamiento de la trama matrimonial}

Hacia principios del siglo XIX, la trama matrimonial, con su conclusión definitiva en uno o más casamientos, era una convención cada vez más recurrente en el género novelístico. Esta estructura encajaba a la perfección con la del Bildungsroman que comenzaba a crecer en popularidad. Al cerrar la historia con un matrimonio convergían el fin de la tensión narrativa, el fin de la juventud y la finalidad de la vida, en un momento histórico en que la trascendencia humana se ligaba con la glorificación de la familia nuclear como célula esencial de la sociedad, en especial para la creciente clase media que era el mercado meta de las novelas. El imperativo teleológico de prosperidad personal y económica sin duda resultaba atractivo cuando el culto al progreso se enraizaba con más fuerza. Esto no significa que el matrimonio como final feliz se utilizara sin reservas. De modo similar a lo que

${ }^{16}$ Las heroínas con frecuencia terminan exiliadas en conventos católicos en Europa continental o en regiones remotas de Gran Bretaña, como Gales. En The British Recluse (1722) de Eliza Haywood, tras narrarse sus respectivos desengaños, dos mujeres terminan viviendo juntas en armonía y amor -aunque el elemento sexual de su relación jamás se hace explícito, probablemente porque, como bien afirma Terry Castle (1993), hasta tiempos recientes, en Occidente, el deseo sexual entre mujeres ha sido "ghosted-or made to seem invisible-by culture itself" (4)-. 
sucedía con los dramaturgos dieciochescos mencionados en páginas anteriores, la fórmula permitía partir de una estructura bien delimitada que desplazara el interés del suspenso con respecto al desenlace hacia la expectativa sobre cuáles serían los obstáculos entre los contrayentes y cómo los sortearían. Era justo en las negociaciones encaminadas al enlace matrimonial donde se podían introducir toda clase de comentarios críticos, con menor o mayor grado de explicitud. Austen, a quien, inclusive sin conocer a profundidad su obra, se le relaciona de inmediato con la trama matrimonial, siempre utiliza esta estructura con un filtro crítico y dicción irónica. Como es bien sabido, la sátira es uno de los motores principales de la narrativa austeniana (Harris, 2017: xvii-xxi). El uso sardónico que hace la autora de su fórmula más frecuente es sintomático del arraigo de la trama matrimonial como estructura narrativa preferida en la novela de su momento. En otras palabras, la familiaridad del público lector con las convenciones de la trama matrimonial, y del contrato sexual que la sustenta, es lo que permite la magistral ironía de la afirmación con que da inicio Pride and Prejudice - "It is a truth universally acknowledged that a single man in possession of a good fortune must be in want of a wife" (Austen, 1994: 5) —, sin duda, uno de los enunciados iniciales más famosos de la literatura anglófona. Esta oración condensa lo que sucede en las seis novelas de esta autora. Se parte del presupuesto tácito que equipara a las uniones matrimoniales de la baja nobleza y la clase terrateniente (gentry) con transacciones económicas para conservar la posición social y la reputación, noción que el resto del texto se dedica a cuestionar, exponiendo su venalidad y los daños afectivos que crea entre contrayentes reales y potenciales. El famoso discurso indirecto libre del que Austen hace uso y perfecciona en su obra en conjunto es lo que permite el distanciamiento e involucramiento simultáneo de la voz narrativa en las situaciones relatadas.

Sin embargo, a final de cuentas, a pesar de la ironía y la sátira, en estas novelas la unión matrimonial idealizada - que liga lo económico y lo afectivo como la base de la felicidad personal y la estabilidad social- termina por realizarse. Así, no obstante las críticas que se hacen al mercantilismo del cortejo, el contrato social y el contrato narrativo se funden en el anuncio del enlace y la promesa de que la pareja fue feliz para siempre. Como apunta Armstrong (1987), en esta clase de 
finales, las reticencias personales se resuelven y las asperezas entre clases y jerarquías sociales se liman: "such a representation creates personal fulfillment where there had been internal conflict and social unity where there had been competing class interests" (51). Pese a la ironía que lo precede, el final matrimonial implica la aquiescencia al orden social establecido. Tras darse el sí en la iglesia y firmar los papeles correspondientes, la mujer toma el apellido del hombre y, de manera simbólica, se vuelven uno mismo. Con esto, quedan atrás las diferencias sociales que en algún momento los distinguían y separaban.

Es en parte a todo esto a lo que novelistas posteriores, como Charlotte y Emily Brönte, reaccionan en sus novelas domésticas. En Fane Eyre, el (des)enlace matrimonial esperado entre la heroína homónima y Rochester no es del todo feliz, al menos en términos convencionales. Una parte de la historia subvierte lo que ocurre en Pamela. A quienes anticipan que el romance que involucra a la empleada (en este caso institutriz y, por ende, de mayor rango que Pamela) desembocará en el reconocimiento por parte del hombre de su amor y la aceptación de un matrimonio por debajo de su rango social, esta novela les ofrece varias decepciones. El primer obstáculo para la unión - la revelación de que Rochester está casado y su esposa, con tendencias pirómanas, ha sido confinada al ático- no es, a fin de cuentas, el de mayor peso. La herencia que recibe Jane hacia el final de la historia es la que le otorga, en palabras de Armstrong (1987), "the power to pursue sexual desires that magically claim priority over any social duty [...]. Only when she no longer needs [Rochester's] money can she become the mistress of his heart, and it is in this role, not as a governess, that she takes her rightful place of dominion over his home" (54). Este giro de la trama resulta una suerte de rebelión en contra de la fórmula austeniana, puesto que la protagonista, de rango social menor, termina por acoger y proteger a quien antes fuera su superior y ahora es un ciego caído en desgracia. Este final agridulce dista de la "felicidad perfecta" - con todos sus bemoles críticos - citada al inicio de este artículo, con que cierra Emma, considerada por algunos como la obra maestra de Austen.

Emily Brönte va aún más allá con su relato de pasión violenta y sexualidad desbordada. En Wuthering Heights, el matrimonio esperado nunca ocurre y, en su 
lugar, suceden uniones desastrosas que impiden cualquier asomo de felicidad para los personajes involucrados. En este caso, el motivo del huérfano de costumbres cuestionables que se enamora de la heredera respetable recuerda a Tom fones. Brönte, sin embargo, se aleja por completo de este modelo y tuerce la trama matrimonial para mostrar la infelicidad que puede acarrear el contrato social que se sobrepone al sexual (algo que, por lo general, ocurre en Austen con consecuencias gratas). Asimismo, la complicada urdimbre de voces y planos narrativos dificulta saber si la celebración del matrimonio subversivo esperado entre Heathcliff y Catherine (Earnshaw) hubiera acarreado la felicidad habitual de la novela doméstica. En otras palabras, dado que los lectores conocen a Heathcliff a través del doble filtro de Nelly Dean y Mr. Lockwood, la narración tiende un velo sobre el nivel de maldad innata o inocencia corrompida de este personaje. Así, Brönte parece tomar la fórmula de la trama matrimonial como base para subvertirla hasta las últimas consecuencias. Todo esto ocurre en un momento en que la novela ya era el modelo literario dominante.

Los argumentos que he presentado en estas páginas no se ofrecen como teoría acabada, sino como una suerte de conjetura informada que, se espera, invite a reflexionar sobre el intrigante fenómeno de las relaciones intergenéricas en el siglo dieciocho y más allá. Si bien el romance, con su focalización en el amor como motor del relato, es uno de los modelos esenciales de los que se desprendió la novela, dado que el teatro era un género literario aún más popular - socorrido por mujeres y hombres de diversos estratos sociales-y, al mismo tiempo, más prestigioso entre la crítica literaria del momento, es importante estudiar su papel en la consolidación de un modo de escritura que en nuestros días es la forma literaria más influyente. Considerar al teatro - al que se relaciona con el ámbito público y que durante mucho tiempo fue prerrogativa masculina en la autoría y actuacióncomo una de los posibles modelos de la novela dieciochesca - a la que siempre se ha ligado tanto a la clase media como a las mujeres - constituye una deliciosa ironía que rinde tributo al siglo que se caracteriza por la explosión de lo irónico. Me permito cerrar estas páginas con una adaptación del célebre ejemplo de E. M. Forster para distinguir entre trama e historia. El horizonte crítico actual nos pre- 
senta una historia: "la comedia teatral (con su trama matrimonial) fue censurada y luego la novela se consolidó como género literario dominante”. En estas páginas yo traté de crear una trama: "la comedia teatral (con su trama matrimonial) fue censurada y la novela, al ver reducida su competencia y tener más escritores que se dedicaran a ella con miras a alcanzar fama y prestigio intelectual, se consolidó como género literario dominante; al hacerlo, echó mano de la estructura narrativa más recurrente de la comedia teatral”. ${ }^{17}$

\section{REFERENGIAS BIBLIOGRÁFICAS}

AN Essay on the New Species of Writing Founded by Mr Fielding. (1751). Londres: W. Owen.

Anderson, Misty. (2002). Female Playwrights and Eighteenth-century Comedy: Marriage on the London Stage. Basingstoke: Palgrave McMillan.

ARAG, Jonathan (ed.). (2019). "Introduction: Desire and Domestic Fiction after Thirty Years". Modern Language Quarterly, 8o(1), 1-5. https://doi.org/10.1215/002679297247204

Armstrong, Nancy. (1987). Desire and Domestic Fiction: A Political History of the Novel. Oxford: Oxford University Press.

Austen, Jane. (1994 [1813]). Pride and Prejudice. Londres: Penguin Popular Classics. Austen, Jane. (2000 [1816]). Emma (Stephen M. Parrish, ed.), Tercera edición. Nueva York: Norton Critical Edition.

BARBAUlD, M. Anna Letitia (ed). (1804). The Correspondence of Samuel Richardson.... Londres: Lewis and Rodem.

BEvIS, Richard W. (1988). English Drama: Restoration and Eighteenth Century, 16601789. Londres: Longman.

${ }^{17}$ La cita original de Forster (1927) es "'The king died and then the queen died' is a story. 'The king died, and then the queen died of grief' is a plot" (86). 
BOONE, Joseph Allen. (1987). Tradition Counter Tradition, Love and the Form of Fiction. Chicago: University of Chicago Press.

CASTlE, Terry. (1993). The Apparitional Lesbian: Female Homosexuality and Modern Culture. Cambridge: Cambridge University Press.

CAStro-SAntAnA, Anaclara. (2018). Errors and Reconciliations: Marriage in the Plays and Novels of Henry Fielding. Nueva York: Routledge.

DoODY, Margaret Anne. (1996). The True Story of the Novel. Nueva Brunswick: Rutgers University Press.

Downie, J. A. (2009). A Political Biography of Henry Fielding. Londres: Pickering and Chatto.

FARQUHAR, George. (1988 [1698]). The Works of George Farquhar (Strum Kenny, ed.), Vol. 1. Oxford: Oxford University Press.

FIELDING, Henry. (1967 [1742]). The Adventures of Foseph Andrew and of His Friend Mr Abraham Adams (Martin Battestin, ed.). Oxford: Clarendon Press.

FIELDING, Henry. (2004 [1730]). “The Author's Farce”. En Henry Fielding, Plays (Thomas Lockwood, ed.), Vol 1. Oxford: Clarendon Press.

FORCEY, B. (2005). “Domestic Fiction”. En The Oxford Companion to Women's Writing in the United States [versión electrónica]. Oxford Reference. Oxford: Oxford University Press. http://doi.org/10.1093/acref/9780195066081.001.0001 FORSTER, E. M. (1927). Aspects of the Novel. Nueva York: Hartcourt. FREEDGOOD, E. (2006). "Domestic Fiction”. En The Oxford Encyclopedia of British Literature [versión electronica]. Oxford Reference. Oxford: Oxford University Press. http://doi.org/10.1093/acref/9780195169218.001.0001

GAY, John. (1983 [1714]). The What D'ye Call It?: A Tragi-comi-pastoral Farce. En John Fuller (ed.), Fohn Gay, Dramatic Works, Vol. 1. Oxford: Clarendon Press.

HARRIS, Jocelyn. (2017). Satire, Celebrity, and Politics in Fane Austen. Lanham: Bucknell University Press.

HumE, Robert. (1988). Henry Fielding and the London Theatre, 1728-173\%. Oxford: Clarendon Press.

HunTER, J. Paul. (1990). Before Novels: The Cultural Contexts of Eighteenth-century English Fiction. Nueva York: Norton. 
KEYMER, Thomas; SABOR, Peter. (2005). Pamela in the Marketplace: Literary Controversy and Print Culture in Eighteenth-century Britain and Ireland. Cambridge: Cambridge University Press.

KINSERVIK, Matthew J. (2002). Disciplining Satire: The Censorship of Satiric Comedy on the Eighteenth-century London Stage. Lewisburg: Bucknell University Press.

LYNCH, Deidre. (2019). "Social, Sexual, and Other Contracts in Eighteenthcentury Novels". Modern Language Quarterly, 8o(1), 21-27. https://doi. org/10.1215/00267929-7247243

MaDermotT, Hubert. (1989). Novel and Romance: The Odyssey to Tom Jones. Basingstoke: Macmillan Press.

MGKEON, Michael. (1987). The Origins of the English Novel: 1600-1740. Baltimore: The Johns Hopkins University Press.

MaKEON, Michael(ed.). (2000). Theory of the Novel: A Historical Approach. Baltimore: The Johns Hopkins University Press.

Michals, Teresa. (2010). “'Like a Spoiled Actress off the Stage': Anti-theatricality, Nature, and the Novel". Studies in Eighteenth-Century Culture, 39, 191-214. http://doi.org/10.1353/sec.0.0057

Milhous, Judith. (2004). “Theatre Companies and Regulation”. En (Joseph Donohue, ed.), The Cambridge History of British Theatre, Vol 2, 1660-1895. Cambridge: Cambridge University Press. 108-125.

Moody, Jane. (2012). "Licensing Acts". En Dennis Kennedy (ed.), The Oxford Companion to Theatre and Performance [versión electrónica]. Oxford Reference. http://doi.org/10.1093/acref/9780199574193.001.0001

O'CONNELL, Lisa. (2011). "Vicars and Squires: Religion and the Rise of the English Marriage Plot”. The Eighteenth-Century, 52(3-4), 383-402.http://doi.org/10.1353/ ecy.2011.0022

RITCHIE, Fiona; SABOR, Peter. (2012). "Introduction”. En Fiona Ritche y Peter Sabor (eds.), Shakespeare in the Eighteenth Century. Cambridge: Cambridge University Press.

ROGERS, Pat. (2010). "Burney [married name D’Arblay], Frances [Fanny]” (en línea). Oxford Dictionary of National Biography. https://doi.org/10.1093/ref:odnb/603 
Ross, I. (2004). "Sheridan [née Chamberlaine], Frances"(en línea). Oxford Dictionary of National Biography. https://doi.org/10.1093/ref:odnb/25365

SPACKS, Patricia Meyers. (2006). Novel Beginnings: Experiments in Eighteenthcentury English Fiction. New Haven: Yale University Press.

StOne, Laurence. (1990). Road to Divorce England 1530-1987, Segunda Edition. Oxford: Oxford University Press.

TANNER, Tony. (1979). Adultery in the Novel: Contract and Transgression. Baltimore: The Johns Hopkins University Press.

TAPLin, Oliver. (2001). "Greek Theatre”. En John Russell Brown (ed.), The Oxford Illustrated History of the Theatre. Oxford: Oxford University Press.

THOMAS, David. (2014). "The 1737 Licensing Act and its Impact”. En Julia Swindells y David Francis Taylor (eds.), The Oxford Handbook of the Georgian Theatre, 1737-1832. Oxford: Oxford University Press.

WARNER, William B. (1998). Licensing Entertainment: The Elevation of Novel Writing in Britain, 1684-1750. Los Angeles: University of California Press. 